\title{
Weniger Parodontitis bei Verwendung von Zahnseide
}

Cepeda MS et al. Association of flossing/ inter-dental cleaning and periodontitis in adults. J Clin Periodontol 2017; 44: 866-871

Die Durchführung einer effektiven häuslichen Mundhygiene wird als wichtiger Präventionspfeiler für die Erhaltung der oralen Gesundheit angesehen. Obwohl die Verwendung von Zahnseide als substanzielle Maßnahme der Mundhygiene erachtet wird, konnten systematische Reviews und Metaanalysen von kontrollierten klinischen Studien nur eine geringe Reduktion von Plaque und Zahnfleischentzündungen bei Benutzung von Zahnseide zeigen. Dabei wurde der Effekt der Anwendung von Zahnseide in klinischen Studien bisher nur an weniger als 600 Probanden untersucht.

In der vorliegenden Querschnittsstudie wurde der Zusammenhang zwischen der Verwendung von Zahnseide und dem Vorliegen einer parodontalen Erkrankung an 6939 Studienteilnehmern der amerikanischen Bevölkerung untersucht.

\section{Methodik}

Die Frequenz der Zahnseideverwendung wurde in folgenden Kategorien erfasst: maximal einmal, zwei- bis viermal oder mindestens fünfmal pro Woche. Die Parodontitis wurde nach den 2007 entwickelten Kriterien der Amerikanischen Gesellschaft für Parodontologie und dem Zentrum für chronische Erkrankungen (AAP-CDC) diagnostiziert. Die Analysen wurden für die möglichen Einflussfaktoren Alter, Geschlecht, Rauch- und Trinkgewohnheiten, Einkommen sowie der Anzahl der jährlichen Zahnarztbesuche korrigiert.

\section{Ergebnisse}

Bei $40 \%$ der Studienteilnehmer wurde Parodontitis festgestellt. Bei mindestens zweimaliger Anwendung der Zahnseide pro Woche waren die Chancen, an einer Parodontitis zu erkranken, um $17 \%$ geringer im Vergleich zu einer selteneren Zahnseideverwendung. Interessanterweise konnte kein dosisabhängiger Zusammen- 
hang zwischen der Frequenz der Zahnseidenutzung pro Woche und der Häufigkeit der Parodontitisdiagnose festgestellt werden. Bei Männern wurde im Vergleich zu Frauen doppelt so häufig Parodontitis festgestellt, Nichtraucher und Probanden mit den höheren Einkommen hatten weniger häufig eine Parodontitis im Vergleich zu Rauchern und Menschen mit geringen Einkommen.

FAZIT

Die Verwendung von Zahnseide zeigte in der vorliegenden Studie einen schützenden Effekt vor parodontalen Erkrankungen. Dabei könnte die interdentale Reinigung mit Zahnseide an zwei bis vier Tagen pro Woche den gleichen schützenden Effekt wie eine häufigere Nutzung haben. Die Autoren führen als möglichen Grund dafür an, dass entzündliche Prozesse im parodontalen Gewebe erst nach mehreren Tagen des Plaquewachstums entstehen. Ein großer Pluspunkt dieser Untersuchung ist die große Studienpopulation mit fast 7000 Teilnehmern, aufgrund des Studiendesigns (Querschnittsstudie/Beobachtungsstudie) ist die Interpretation eines kausalen Zusammenhangs jedoch nicht möglich.

Korrespondenzadresse

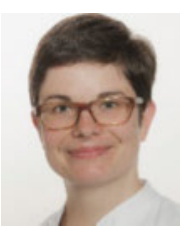

\section{Dr. Daniela Hoedke}

Abteilung für Parodontologie und Synoptische Zahnmedizin Charité - Universitätsmedizin Berlin

daniela.hoedke@charite.de 